\title{
Analysis on Boundary Conditions for Elastic Structures
}

\author{
WX Zhang ${ }^{1, *}$ \\ ${ }^{1}$ Nantong Polytechic College, Nantong, Jiangsu, 226002, China
}

\begin{abstract}
With the rapid development of modern industry, elastic materials and structures have been widely used in all walks of life, such as construction, machinery and so on. The traditional elastic mechanics method generally adopts the semi inverse method. However, this method ignores the local deformation caused by boundary constraints and can only find part of the solution of the problem. In this paper, the basic equations of symplectic system are established, and the solutions of various boundary condition problems are given.
\end{abstract}

\section{Introduction}

In mechanical calculation, people usually only care about the effect of load on the whole material and structure, and the local effect is often ignored. For some special cases, such as the bending cantilever beam, the stress near the fixed end is not uniformly distributed, and the stress at the upper and lower corners is far greater than the average stress on the section [1-3]. Therefore, due to the diversity of mechanical boundary conditions, the traditional elastic mechanics calculation method can not obtain ideal numerical results in a large number of problems, and the new solution method has attracted much attention [4-6].

$$
\varepsilon_{p}(u, w)=\frac{1}{2}\left\{C_{11}\left(\partial_{x} u\right)^{2}+C_{33} \dot{w}^{2}+C_{44}\left[\dot{u}^{2}+\left(\partial_{x} w\right)^{2}\right]\right\}+C_{44} \dot{u} \partial_{x} w+C_{13} \partial_{x} u \dot{w}
$$

After theoretical derivation, we establish the basic equation of dual form, which is expressed as follows

$$
\begin{aligned}
& \dot{u}=a_{1} p_{1}-\frac{\partial w}{\partial x} \\
& \dot{w}=a_{3} p_{2}-a_{2} \frac{\partial u}{\partial x} \\
& \dot{\tau}_{x z}=C_{44} \dot{u}+C_{44} \frac{\partial w}{\partial x} \\
& \dot{\sigma}_{z}=C_{13} \frac{\partial u}{\partial x}+C_{33} \dot{w}
\end{aligned}
$$

Its charateristic equation is
Single variable method is usually used in traditional numerical calculation. This method can reduce the dimension of the equation, but can not avoid the difficulty of solving higher order partial differential equations [7]. In this paper, based on symplectic system, the solution of mechanical problems of elastic structures boundary conditions are discussed.

\section{Solution method}

Ignoring the effect of volume force, the Lagrange function can be expressed as follows: is studied, and the treatment methods of various 


$$
\begin{aligned}
& \bar{u}=M_{1 j}\left(C_{j} J_{n-1}+C_{j+3} Y_{n-1}\right)+N_{1 j}\left(C_{j} J_{n}+C_{j+3} Y_{n}\right) \\
& \bar{v}=M_{2 j}\left(C_{j} J_{n-1}+C_{j+3} Y_{n-1}\right)+N_{2 j}\left(C_{j} J_{n}+C_{j+3} Y_{n}\right) \\
& \bar{p}_{1}=M_{4 j}\left(C_{j} J_{n-1}+C_{j+3} Y_{n-1}\right)+N_{4 j}\left(C_{j} J_{n}+C_{j+3} Y_{n}\right) \\
& \bar{p}_{2}=M_{5 j}\left(C_{j} J_{n-1}+C_{j+3} Y_{n-1}\right)+N_{5 j}\left(C_{j} J_{n}+C_{j+3} Y_{n}\right)
\end{aligned}
$$

\section{Numerical example}

According to the numerical calculation, we can obtain all the eigenvalues and the corresponding eigensolutions.
These eigensolutions not only describe the basic mechanical deformation such as tension and torsion, but also describe local effects caused by boundary constraints and temperature conditions. but also describe the local effects caused by boundary constraints and temperature conditions which cannot be obtained by traditional methods.

Fig. 1 and Fig. 2 below show the numerical results obtained by the above method. From these figures, there is a close relationship between eigenvalues and material constants. Fig. 3 exhibits the relaxation property of the solid.

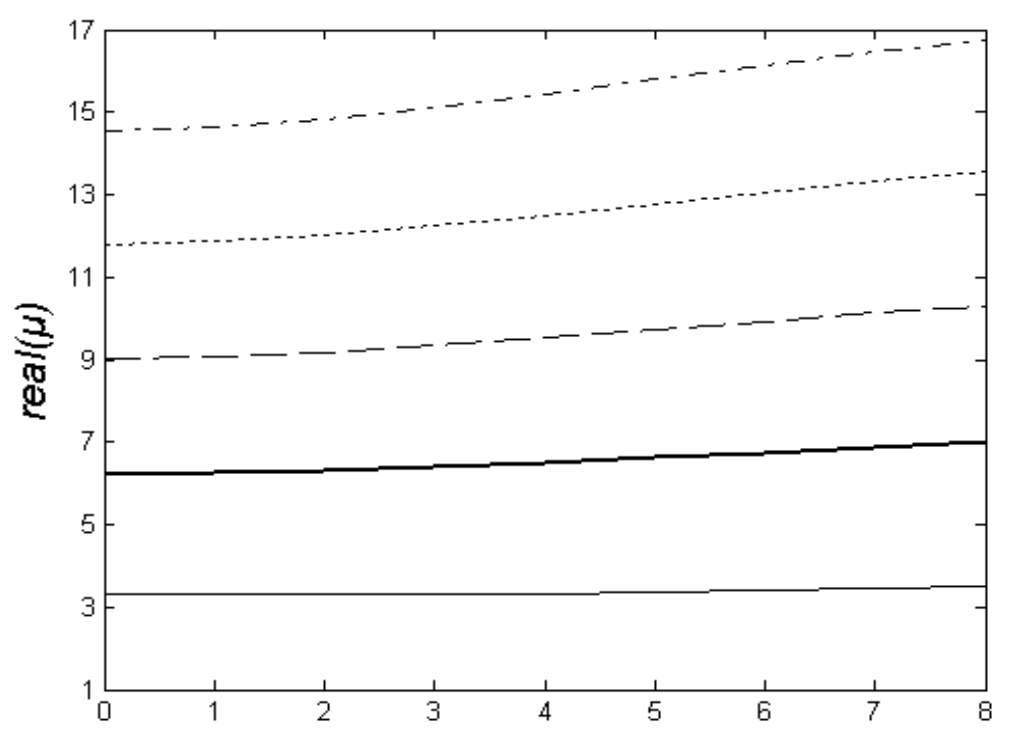

Figure 1. The stress distribution

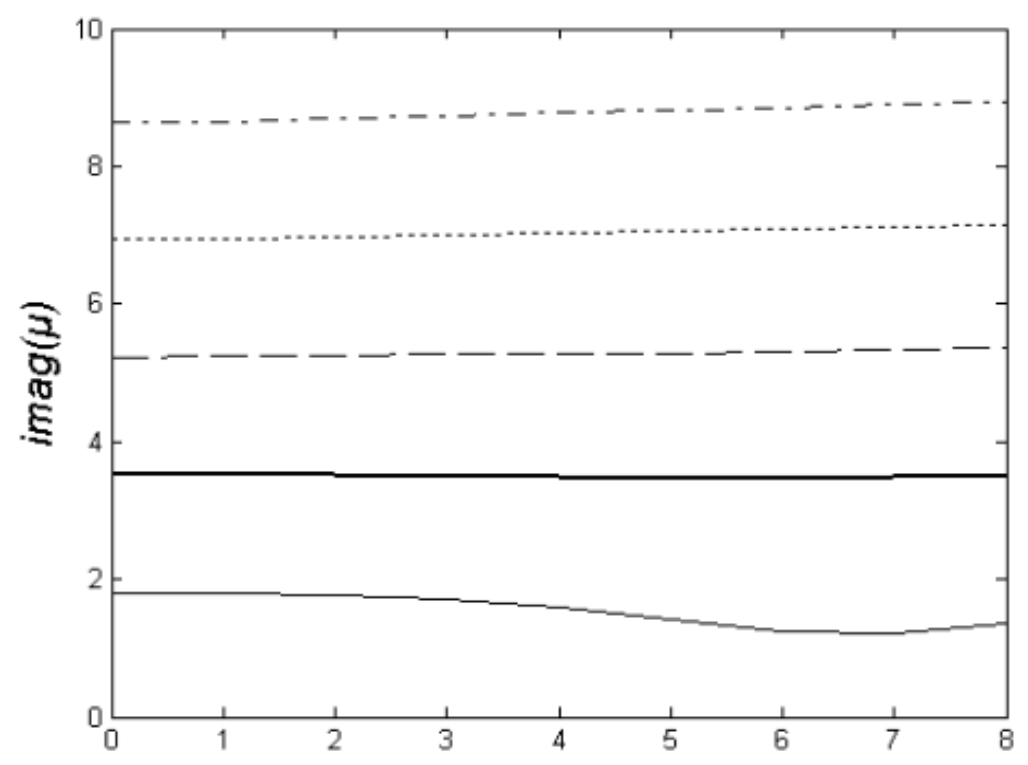

Figure 2. The strain distribution 


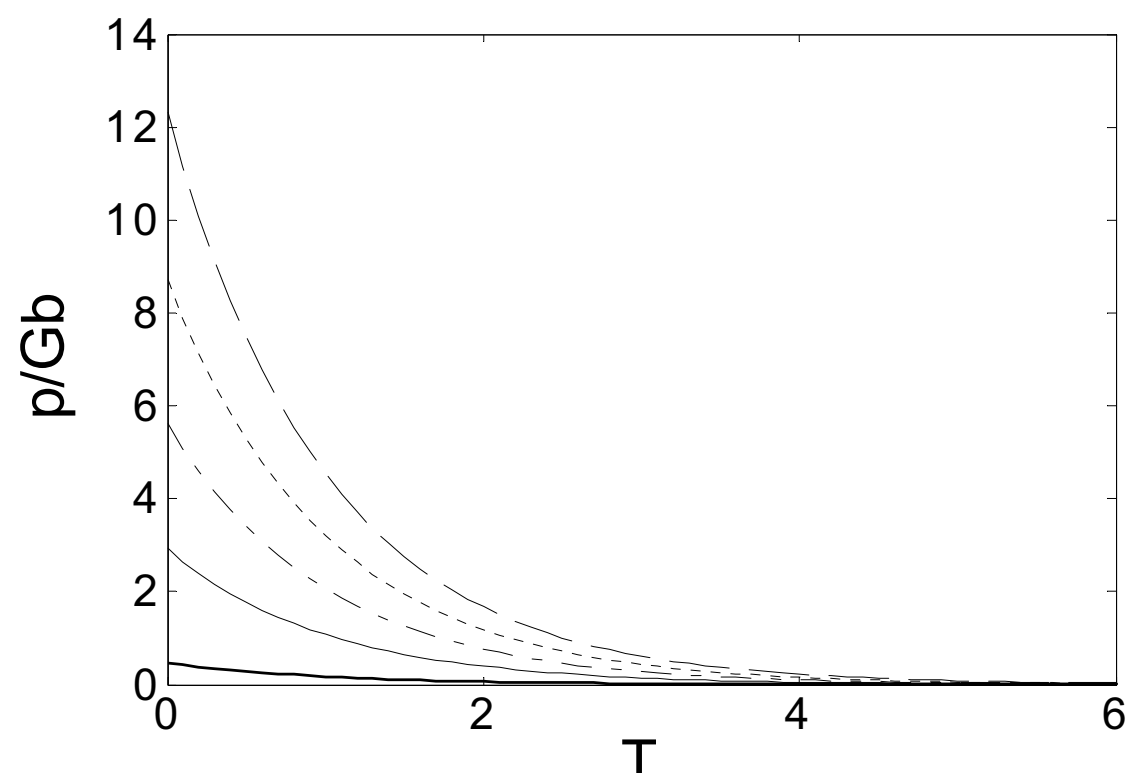

Figure 3. Relaxation of stress

\section{Conclusion}

7. Berdichevsky, V.L. (2006) On thermodynamics of crystal plasticity. Scripta Mater., 54: 711-716.

Symplectic geometry, algebraic geometry and differential geometry are three parallel branches of mathematics, which study the geometric and topological properties of symplectic manifolds. The origin of the Symplectic geometry is closely related to classical mechanics in physics, and also has a very important connection with algebra, geometry, mathematical physics and other fields in mathematics. In this paper, the conventional problem of transversely isotropic elasticity is discussed.

\section{Reference}

1. Govorukha, V.B., Loboda, V.V. (2000) Contact zone models for an interface crack in a piezoelectric material. Acta Mech., 140: 233-246.

2. Govorukha, V.B., Munz, D., Kamlah, M. (2000) On the singular integral equations approach to the interface crack problem for piezoelectric materials. Arch Mech., 52: 247-273.

3. Meraghni, F., Desrumaux, F., Benzeggagh, M.L. (1999) Mechanical behaviour of cellular core for structural sandwich panels. Composites A, 30: 767779.

4. Laudiero, F., Zaccaria, D. (1988) A consistent approach to linear stability of thin-walled beams of open sections. Int. J. Mech. Sci., 30: 543-557.

5. Ortiz, M., Repetto, E.A. (1999) Nonconvex energy minimization and dislocation structures in ductile single crystals. J. Mech. Phys. Solids, 47: 397-462.

6. Berdichevsky, V.L. (2005) Homogenization in micro-plasticity. J. Mech. Phys. Solids, 53: $2457-$ 2469. 